\title{
Interplay of crystal thickness and in-plane anisotropy and evolution of quasi-one- dimensional electronic character in
}

Hart, Lewis S.; Gunasekera, Surani M.; Mucha-Kruczyski, Marcin; Webb, James L.; Avila, José; Asensio, María C.; Wolverson, Daniel

Published in:

Physical Review B

Link to article, DOI:

10.1103/PhysRevB.104.035421

Publication date:

2021

Document Version

Publisher's PDF, also known as Version of record

Link back to DTU Orbit

Citation (APA):

Hart, L. S., Gunasekera, S. M., Mucha-Kruczyski, M., Webb, J. L., Avila, J., Asensio, M. C., \& Wolverson, D. (2021). Interplay of crystal thickness and in-plane anisotropy and evolution of quasi-one-dimensional electronic character in. Physical Review B, 104(3), [035421]. https://doi.org/10.1103/PhysRevB.104.035421

\section{General rights}

Copyright and moral rights for the publications made accessible in the public portal are retained by the authors and/or other copyright owners and it is a condition of accessing publications that users recognise and abide by the legal requirements associated with these rights.

- Users may download and print one copy of any publication from the public portal for the purpose of private study or research.

- You may not further distribute the material or use it for any profit-making activity or commercial gain

- You may freely distribute the URL identifying the publication in the public portal 


\title{
Interplay of crystal thickness and in-plane anisotropy and evolution of quasi-one-dimensional electronic character in $\mathbf{R e S e}_{2}$
}

\author{
Lewis S. Hart, ${ }^{1}$ Surani M. Gunasekera, ${ }^{1}$ Marcin Mucha-Kruczyński ${ }^{\circ},{ }^{1}$ James L. Webb, ${ }^{2}$ José Avila, ${ }^{3}$ \\ María C. Asensio, ${ }^{4}$ and Daniel Wolverson $\mathbb{1}^{1, *}$ \\ ${ }^{1}$ Centre for Nanoscience and Nanotechnology and Department of Physics, University of Bath, Bath BA2 7AY, United Kingdom \\ ${ }^{2}$ Center for Macroscopic Quantum States (bigQ), Department of Physics, Technical University of Denmark, 2800 Kongens Lyngby, Denmark \\ ${ }^{3}$ Synchrotron SOLEIL, Saint Aubin and Université Paris-Saclay, BP 4891192 Gif-sur-Yvette, France \\ ${ }^{4}$ Madrid Institute of Materials Science (ICMM), Spanish Scientific Research Council (CSIC) and CSIC associated unit MATINÉE (ICMM and \\ Institute of Materials Science of Valencia University, ICMUV) Cantoblanco, E-28049 Madrid, Spain
}

(Received 25 January 2021; revised 4 June 2021; accepted 25 June 2021; published 15 July 2021)

\begin{abstract}
We study the valence band structure of $\mathrm{ReSe}_{2}$ crystals with varying thickness down to a single layer using nanoscale angle-resolved photoemission spectroscopy and density functional theory. The width of the top valence band in the direction perpendicular to the rhenium chains decreases with decreasing number of layers, from $\sim 200 \mathrm{meV}$ for the bulk to $\sim 80 \mathrm{meV}$ for monolayer. This demonstrates increase of in-plane anisotropy induced by changes in the interlayer coupling and suggests progressively more one-dimensional character of electronic states in few-layer rhenium dichalcogenides.
\end{abstract}

DOI: 10.1103/PhysRevB.104.035421

\section{INTRODUCTION}

The transition metal dichalcogenides (TMDs) present a fascinating diversity of behavior including: thicknessdriven indirect-to-direct semiconducting band gap transitions; metals; magnetic phases; charge density wave materials; superconductors, and materials with topological surface states [1]. They are readily combined in vertical heterostructures with atomically precise interfaces and offer unprecedented degrees of built-in control over their optical [2] and electronic [3] properties via novel means such as variation of the heterostructure twist angle [4], and more conventional methods such as strain [5] or electrostatic gating [6].

Within the TMD family, $\mathrm{ReSe}_{2}$ and $\mathrm{ReS}_{2}$ are distinguished by in-plane anisotropy due to a Jahn-Teller distortion, which breaks the $120^{\circ}$ in-plane rotational symmetry common to twodimensional crystals and leads to the formation of rhenium chains [7]. It also causes out-of-plane buckling of chalcogens, which results in lack of atomic registry between consecutive layers and contributes to weakening of the interlayer interaction. The in-plane anisotropy and weak interlayer coupling impact many of the properties of rhenium dichalcogenides, from charge transport and optical absorption [8] to high catalytic activity as shown in solar water splitting [9-11].

One of the most intriguing fundamental questions associated with layered materials is the understanding of the changes occurring as they are thinned down from threedimensional bulk to single two-dimensional atomic planes. In many materials such as $2 \mathrm{H}$ semiconducting TMDs [12-14], post-transition-metal monochalcogenides [15-17] and black phosphorus [18], parts of the valence band are formed by orbitals extending significantly in the out-of-plane direction (e.g., $p_{z}$ or $d_{z^{2}}$ ) which are strongly affected by the presence

*d.wolverson@bath.ac.uk of neighboring layers and interlayer coupling. As a result, the shape of the valence band depends sensitively on the number of layers in the crystal [19] leading to, for example, indirect-to-direct band-gap transitions [12-14]. In contrast, in rhenium TMDs, it is rhenium $d_{x y}$ and $d_{x^{2}-y^{2}}$ rather than $d_{z^{2}}$ or chalcogen $p_{z}$ orbitals that form the top of the valence band [20,21]. As these are the orbitals participating in the Jahn-Teller distortion, this poses the question of whether the in-plane anisotropy of the valence band could be influenced by tuning the interlayer interaction.

Here, we use nanoscale angle-resolved photoemission spectroscopy (nano-ARPES) supported by density functional theory calculations to study the interplay between the in-plane anisotropy and crystal thickness in the electronic band structure of $\mathrm{ReSe}_{2}$, from bulk down to the monolayer limit. We show that, as previously argued for this compound [21], the position of the valence band maximum does not change with the number of layers. However, we observe a decrease in the width of the top valence band in the direction perpendicular to the rhenium chains with decreasing thickness, indicating the increasing decoupling of rhenium chains and the growing one-dimensional character of the electronic states.

\section{METHODS}

Sample preparation, characterisation details, and all relevant raw data are given in Secs. S1, S2, and S3, respectively, within the Supplemental Material (SM) [22]. Samples were prepared by deterministic transfer from bulk crystals [23]. Nano-ARPES data were obtained at the ANTARES beam line of the SOLEIL Synchrotron, Paris, which is equipped with a zone plate allowing a spot size of $120 \mathrm{~nm}$, an angular resolution of $\sim 0.2^{\circ}$ and an energy resolution of $\sim 10 \mathrm{meV}$. The high spatial resolution allows one to map the photoemission signal as a function of position with submicron resolution 
and thus to select flakes of different thickness. In this paper, photon energies of $100 \mathrm{eV}$ were used since the performance of the zone plate is optimal at this energy; the photoionisation cross sections of Re $5 d$ and $\mathrm{Se} 4 p$ at $100 \mathrm{eV}$ are comparable and are of order 0.1 to 0.2 Mbarn [24]. For the bulk flakes, this photon energy probes states with in-plane momenta lying in a plane near the $Z$ point at the top of the Brillouin zone [25]. The sample cleaning process (see Supplemental Material [22]) resulted in layers, which showed atomically clean and flat regions between the bubbles of trapped contaminants; no drift due to charging effects was observed. Raman spectroscopy, core-level nanoscale scanning photoemission microscopy (nano-XPS), and atomic force microscopy (AFM) were used as characterization tools.

Valence-band structures were calculated using firstprinciples plane wave, pseudopotential-based methods within density functional theory (DFT) as implemented in the Quantum Espresso package [26]. Both local density (LDA) and generalised gradient (GGA) approximations for the exchange-correlation functionals were used with the projector augmented wave (PAW) method [27,28] being used in all cases. The valence of Re was taken as 15 (configuration $\left.5 s^{2} 5 p^{6} 5 d^{5} 6 s^{2}\right) .8 \times 8 \times 1$ Monkhorst-Pack [29] $k$-point meshes were used for monolayers, bilayers, and trilayers (12, 24 , and 36 atoms per unit cell, respectively), and at least $6 \times 6 \times 6$ were used for the bulk (12 atoms). Kinetic energy cutoffs were, typically, $>800 \mathrm{eV}$. More computational details are given elsewhere [30,31] where results using scalarand fully-relativisitic pseudopotentials are compared. Here, scalar-relativistic pseudopotentials were chosen in order to obtain wave-function projections onto atomic states classified only by orbital angular momentum (i.e, without spin). Comparisons of calculated 1L band structures with and without spin-orbit coupling (SOC) have already been reported and the features of interest here are still present when SOC is included $[30,32,33]$.

For the band-structure calculations, the unit-cell parameters and atomic positions were taken from experimental data [7] and were relaxed to obtain residual forces generally below $0.01 \mathrm{eV}^{-1}$. All unit-cell parameters were allowed to vary, including the separation between neighboring layers in the bilayer, trilayer, and bulk cases. Representative calculations are shown in Sec. S4 within the Supplemental Material [22] for monolayer, bilayer, trilayer, and bulk band structures in the $\Gamma-M$ direction [22]. Our earlier calculations [25] used the LDA with fully-relativistic pseudopotentials and gave good agreement with ARPES and crystallographic data for bulk $\mathrm{ReSe}_{2}$ despite not taking interlayer van der Waals interactions into account explicitly; in Sec. S4 within the Supplemental Material [22], we also show calculations of the band structure of a bilayer in the $\Gamma-M$ direction, comparing GGA results without van der Waals corrections and with two Grimme-type dispersion corrections, GGA+D2 and GGA+D3 [34].

\section{RESULTS}

\section{A. Characterization of atomically thin $\mathrm{ReSe}_{2}$}

Studying the dependence of the physical properties of layered materials as a function of their thickness requires
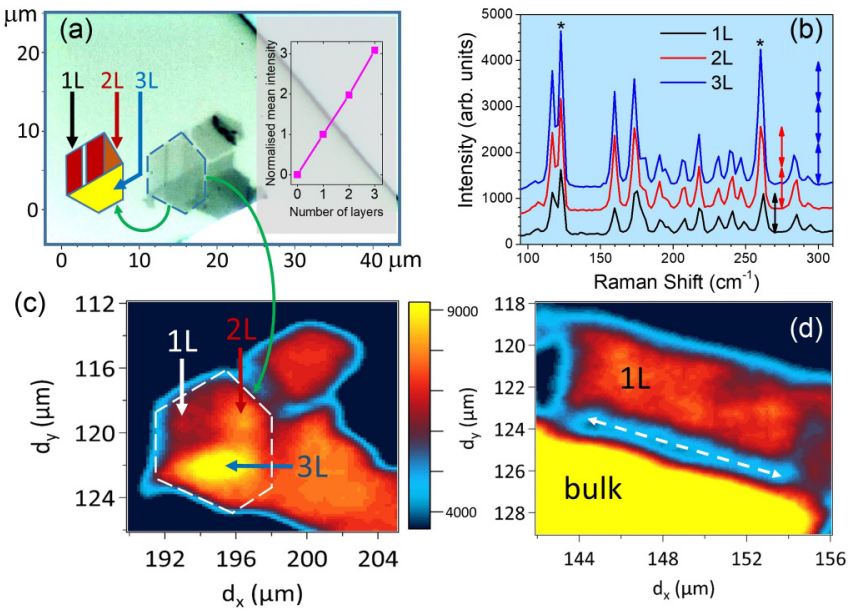

FIG. 1. Structure of the samples investigated. (a) Optical microscopy image of a few-layer $\mathrm{ReSe}_{2}$ flake on an HOPG platform with the region of interest bounded by the dashed blue border; the sketch of this (on the left) identifies mono-, bi-, and tri-layer areas (marked 1L, 2L, 3L, respectively) and the inset shows the dependence of the Raman intensity (averaged over two prominent peaks) on the number of layers; (b) micro-Raman spectra of the regions of the sample shown in (a), with asterisks indicating the two peaks used to calculate the mean intensity. A vertical shift has been applied for clarity; (c) nano-XPS map of the intensity of the Re $4 f$ core levels (binding energy 40-44 eV) of the sample shown in (a) with the same region of interest indicated by the white dashed line; (d) nano-XPS intensity map of $\operatorname{Re} 4 f$ core levels for a second sample with large monolayer and bulk-like regions; the dashed arrow shows a cleavage direction of this flake.

samples with well-established layer numbers down to one monolayer. This task is more challenging than usual for the rhenium-based TMDs since, to enable comparisons between different flakes, their crystallographic orientations must be established including, possibly, their vertical orientation $[35,36]$ as turning a flake upside down is not a symmetry operation for $\mathrm{ReSe}_{2}$. We have overcome this problem by exfoliating large flakes which contain regions of different thicknesses as shown in Fig. 1 (see also Figs. S1 and S2 within the Supplemental Material [22]). Figure 1(a) shows an optical micrograph of a sample whose leftmost flake contains monolayer (1L), bilayer (2L), and trilayer (3L) regions, identified on the sketch to the left of the flake. All the regions derive from the same parent crystal and have preserved the same orientation as implied by the visible cleavage edges [37] at $\sim 60^{\circ}$ and $\sim 120^{\circ}$ and as confirmed rigorously by the Raman spectra of Fig. 1(b). Any significant rotation of one region with respect to the others would result in changes in the relative intensities of the different peaks within the spectrum due to the pronounced in-plane anisotropy of $\operatorname{ReSe}_{2}[30,35,36,38]$. The orientation of the flakes is supported by a reciprocal space map of photoemission intensity for the monolayer, shown in Fig. S3 (within the Supplemental Material [22]), which was recorded near the energy of the valence-band maximum and displays the characteristic anisotropy already known from bulk $\mathrm{ReSe}_{2}$. We confirmed layer numbers by four independent means: Raman spectroscopy, scanning photoemission microscopy, AFM, and nano-ARPES (AFM images and line scans for all samples 
are shown in Figs. S1 and S2 within the Supplemental Material [22]). AFM step height measurements gave a monolayer thickness of $d \sim 0.60$ to $0.67 \mathrm{~nm}$, comparable to the interlayer lattice parameter $c=0.6702 \mathrm{~nm}[7,39]$ (note, the crystallographic $c$ axis in $\operatorname{ReSe}_{2}$ is tilted away from the normal to the layer). The thicknesses of the $2 \mathrm{~L}$ and $3 \mathrm{~L}$ regions were likewise confirmed by AFM (Fig. S2 within the Supplemental Material [22]). Moreover, the Raman intensity from these regions scales linearly with the number of layers. This is best seen by looking at two Raman bands marked with asterisks in Fig. 1(b), which dominate the spectrum and do not overlap strongly with other spectral features-vertical arrows next to the peaks clearly indicate the scaling. We also fit and average areas of these peaks following subtraction of a small background and present the results in the inset of panel (a). Since the silicon substrate does not have a thick oxide layer, and because the $\mathrm{ReSe}_{2}$ layers were placed on a highly oriented pyrolytic graphite (HOPG) flake on the substrate, there is no modulation of the Raman intensity due to interference effects $[30,40]$. The shifts in zone center phonon frequencies with the number of layers are smaller for $\mathrm{ReSe}_{2}$ and $\mathrm{ReS}_{2}$ than for most TMDs [30,41] so that, here, interferencefree Raman intensities are more reliable than Raman peak shifts for determination of layer thicknesses. However, the measured Raman peak positions support our thickness assignments: the bulk modes at 283.6 and $260.5 \mathrm{~cm}^{-1}$ show a small shift to 285.2 and $263.0 \mathrm{~cm}^{-1}$ in the monolayer sample, in agreement with previous experimental and computational results [32].

Figures 1(c) and 1(d) show maps of the photoemission intensity from the $\operatorname{Re} 4 f$ core levels (binding energy $40-44 \mathrm{eV}$ ) from the sample of Fig. 1(a) and one other sample containing monolayer and bulk regions with the same orientation. These maps were recorded primarily to locate the different regions for the ARPES experiments. Interestingly, the contrast in photoelectron count from $1 \mathrm{~L}$ to $3 \mathrm{~L}$ regions reflects the layer thicknesses. The core level photoemission intensity need not necessarily depend linearly on the number of $\mathrm{ReSe}_{2}$ layers, but in principle it could be determined quantitatively by using x-ray photoelectron spectroscopy tools [42]. As Fig. 1(c) shows, discrimination between regions differing by one layer is possible and this mapping correlates with the Raman data. Imaging based on possible shifts in the energy of the $\mathrm{Re}$ $4 f$ core levels [43], on the other hand, did not produce any contrast between the different regions.

\section{B. ARPES results from atomically thin $\mathrm{ReSe}_{2}$}

Because $\mathrm{ReSe}_{2}$ possesses only inversion symmetry, the Brillouin zone of the monolayer is a distorted regular hexagon and the $K$ and $M$ points form three nonequivalent pairs ( $K_{1}, K_{2}, K_{3}, M_{1}, M_{2}, M_{3}$, and their inversion equivalents). In this paper, we consider only dispersions in two key directions: along, and normal to, the Re chains, for simplicity labeled $\Gamma-$ $K$ and $\Gamma-M$ respectively; see Fig. 2(a) for the indication of these directions in the real and reciprocal spaces and Fig. 2(b) for comparison of the bulk and two-dimensional Brillouin zones of $\mathrm{ReSe}_{2}$ (the latter is a projection of the former along the reciprocal space $c^{*}$ axis). These special directions correspond to, for example, the polarization directions of the two band-edge excitons in $\mathrm{ReSe}_{2}$ [44-46] and were identified as the directions of maximum and minimum dispersion in the studies of bulk material $[25,47]$. We present the measured valence-band dispersions for the monolayer (1L) flake shown in Fig. 1(d), in Figs. 2(c) and 2(e) for $\Gamma-M$, and Figs. 2(d) and 2(f) for $\Gamma-K$. The right-hand panels in (c) and (d) show second derivatives with respect to energy of the data in the left, and in (e) and (f) we show expanded views of the same data and its second derivatives close to the valence-band maximum (within $\sim 2 \mathrm{eV}$ ). The extraordinarily flat nature of the top of the valence band in the $\Gamma-M$ direction is striking and is the main result of this paper [Figs. 2(c) and 2(e)]. As we show later, this is very different to the bulk valence-band structure. By contrast, the top valence band remains dispersive in the near-orthogonal direction $\Gamma-K$ [Figs. 2(d) and 2(f)]. Because second derivatives can occasionally generate spurious features, we have examined the energy distribution curves (EDCs), which are the raw data for Fig. 2, directly. Figure S4 within the Supplemental Material [22] shows these EDCs in the $\Gamma-M$ and $\Gamma-K$ directions and confirms the flatness of the band along $\Gamma-M$ while the $\Gamma-K$ direction shows significant dispersion.

One more prominent feature of the $1 \mathrm{~L} \mathrm{ReSe}_{2}$ valence-band structure is the locally flat band centered at $\Gamma$ at about $1 \mathrm{eV}$ below the valence-band edge (at a binding energy of $\sim 2 \mathrm{eV}$ ), which yields a particularly high photoemission intensity. This feature was shown to correspond to the valence band formed predominantly by the out-of-plane $\operatorname{Re} d_{z^{2}}$ and $\operatorname{Se} p_{z}$ orbitals [21]. Figure 2 also shows the valence bands of the HOPG substrate on which the $\mathrm{ReSe}_{2}$ flakes were placed. The observation of the $\Gamma$ point of graphite (at $k_{\|}=0$ ) usefully confirms the experimental location of the first Brillouin zone and $\Gamma$ point of the $\mathrm{ReSe}_{2}$ flakes and, as a result of the relatively large unit cell of $\mathrm{ReSe}_{2}$, the accessible momentum range allows us to sample almost two complete Brillouin zones in the $\Gamma-M$ direction. The data of Fig. 2 show no evidence for hybridization or appreciable opening of gaps at the binding energy and momentum values where the bands of $\mathrm{ReSe}_{2}$ and HOPG intersect. These results contrast with recently reported findings of strong coupling to graphene for TMDs such as $\mathrm{WSe}_{2}$ [48] and $\mathrm{MoS}_{2}$ [49], although, in those studies, the flakes also had graphene capping layers, which the present samples did not.

\section{Evolution of the valence band with the number of layers}

Following the discussion of the ARPES of the monolayer region shown in Fig. 1(d), we move to the flake presented in Figs. 1(a) and 1(c). Between the two samples, we can not only test the reproducibility of the $1 \mathrm{~L}$ results of Fig. 2 as well as those for the bulk, but also study the evolution of the valence-band states as a function of crystal thickness. In Fig. 3, we show photointensity as a function of binding energy and momentum, (a)-(c), and its second-derivative, (d)(f), recorded using nano-ARPES for all our few-layer flakes as well as bulk, (g) and (h), for a momentum slice in the $\Gamma-M$ direction [note that the data in (c) and (f) was not recorded over the same binding energy range as in the other panels]. For this comparison, we labeled the bands of the bulk flake following the high-symmetry points of the projected 2D 

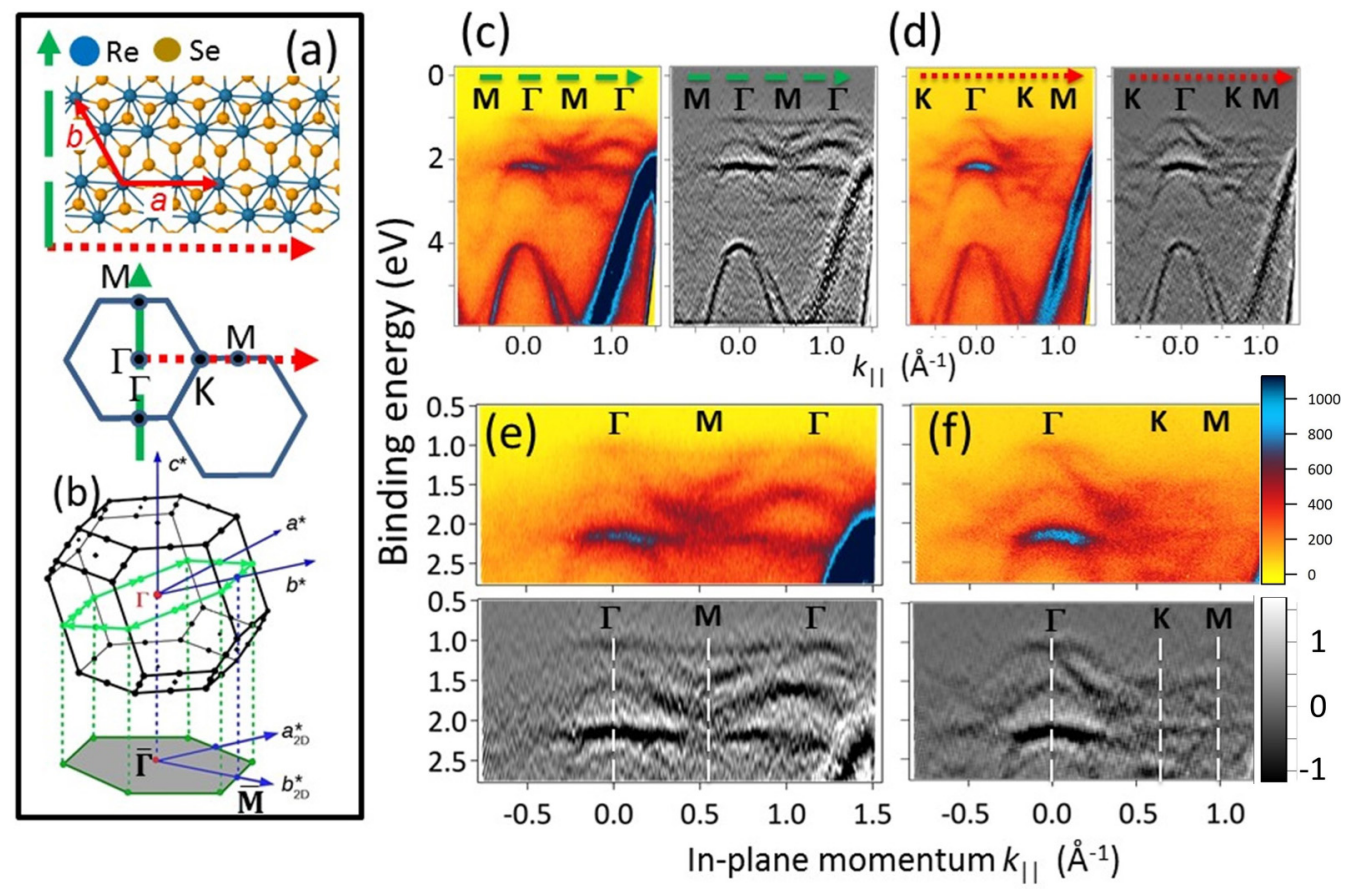

FIG. 2. Valence bands of monolayer $\mathrm{ReSe}_{2}$. (a) Crystal structure (top) and reciprocal lattice of monolayer $\mathrm{ReSe}_{2}$. Solid red arrows indicate in-plane lattice vectors $a$ and $b$; the rhenium "chains" are oriented along $a$. In-plane directions $\Gamma-K$ (red dotted arrow) and $\Gamma-M$ (green dashed arrow) are displayed in relation to the top view of the real space layer plane. (b) First Brillouin zone of bulk ReSe $e_{2}$ with reciprocal lattice vectors $a^{*}, b^{*}$, and $c^{*}$ and their projections onto the 2D layer plane; $\Gamma$ and $Z$ points are also shown. (c) Left: photoemission intensity (false color) as a function of binding energy and in-plane wave vector for the direction $\Gamma-M$ indicated by the green dashed arrow defined in (a). The intense dispersive bands at binding energies of $2 \mathrm{eV}$ and greater originate from the graphite substrate. Right: second derivatives of the same data. (d) Left: photoemission intensity as in (a) but for the in-plane wave vector in the direction $\Gamma-K$, which is close to orthogonal to the direction of (a) and which is indicated by the red dotted arrow in (a). Right: second derivatives of the same data. [(e),(f)] Expanded views of the raw and second-derivative data of (c) and (d), respectively, near the valence-band edge.

Brillouin zone, using symbols $\bar{\Gamma}, \bar{M}$ and $\bar{K}$ to indicate that the vertical momentum component of the bands is not specified [at the photon energy used here $(100 \mathrm{eV})$, ARPES probes states lying in an approximately planar surface passing near the $Z$ point at the top of the 3D Brillouin zone [25] shown in Fig. 2(b), i.e, $\bar{\Gamma} \approx Z$ ]. Although the data for monolayer are not as clear as those obtained with the larger sample of Fig. 2, there is a systematic trend in the bandwidth of the uppermost valence band.

We use our raw data to obtain the width of that band-we extract photoemission counts as a function of binding energy (that is, the energy distribution curves, EDCs) and fit these to obtain a measure of the band-edge position at each momentum along the $\Gamma-M$ and $\Gamma-K$ directions. This, in turn, allows us to identify the energies of the band extrema. The results of our procedure (for further details of the fitting process see the SM, Figs. S5 and S6 [22]) are shown in Fig. 4(a), where we compare the extracted top valence bands for $1 \mathrm{~L}, 2 \mathrm{~L}, 3 \mathrm{~L}$, and bulk. The curves for $2 \mathrm{~L}, 3 \mathrm{~L}$, and bulk are shifted vertically by steps of $0.4 \mathrm{eV}$ for clarity (the same shift is applied to $\Gamma-M$ and $\Gamma-K$ in each case). The corresponding raw data are shown in the SM, Figs. S7- S9 [22] (in Fig. S11 we show a comparison of the raw data with the calculated band structures of bulk and monolayer samples in the $\Gamma-K$ and $\Gamma-M$ directions). A summary of the bandwidths obtained from fitting the EDCs is displayed in Fig. 4(b), which illustrates flattening of the top valence band along $\Gamma-M$ with decreasing crystal thickness while the bandwidth along $\Gamma-K$ is comparable for three out of four investigated sample thicknesses. The error bars are estimated from the sensitivity of the measured bandwidth to variation of the fitting parameters (principally the threshold intensity $I_{\text {thresh }}$ corresponding to the band edge; see Fig. S6 for a definition of $I_{\text {thresh }}$ ). The error for the case of the bilayer in the $\Gamma-K$ direction is somewhat larger as the data is slightly poorer (see Fig. S8 within the Supplemental Material [22]); the $2 \mathrm{~L}$ region was the smallest of the flakes [see Fig. 1(a)] and therefore the most difficult to measure. Note, bandwidth values in the bulk case are photon energy-dependent due to the dispersion normal to the layers; our present values agree well with previous values measured on cleaner, cleaved bulk material [25] at the same photon energy.

Our experimental findings are also supported by density functional theory (DFT) calculations. Using scalar relativistic pseudopotentials, we obtained bandwidths of $95 \mathrm{meV}$ (1L), $150 \mathrm{meV}(2 \mathrm{~L}), 275 \mathrm{meV}$ (3L), and $500 \mathrm{meV}$ for bulk. A fully relativistic calculation yielded similar values of $85,187,217$, and $436 \mathrm{meV}$, respectively. The calculated band structures along the $\Gamma-M$ direction are shown in Sec. S4 within the Supplemental Material(Fig. S10) [22] and the trend in their variation as a function of crystal thickness agrees well with the measurements.

At the photon energy of $100 \mathrm{eV}$ used here for the nano-ARPES experiments, the recorded signal is highly surface-specific: the predicted inelastic mean free path of 

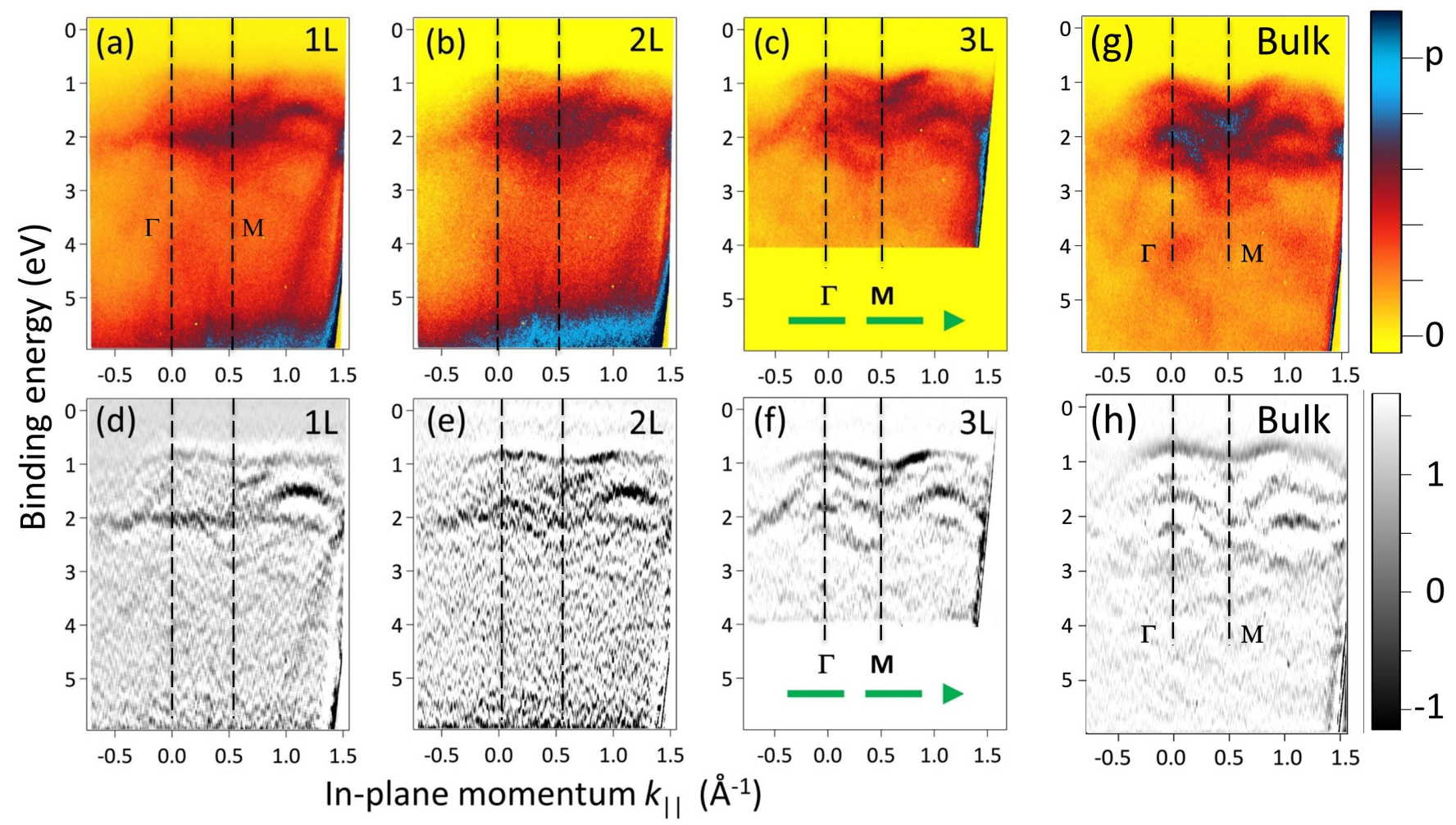

FIG. 3. Comparison of the measured $\mathrm{ReSe}_{2}$ valence-band dispersion along $\Gamma-M$ (indicated by the green dashed arrow defined in Fig. 1) for mono-, bi-, and tri-layer flakes (labeled 1L, 2L, and 3L respectively) and bulk. [(a)-(c),(g)] ARPES data; [(d)-(f),(h)] second derivatives of the data above. Vertical dashed lines indicate the positions of the $\Gamma$ and $M$ points on each panel. The color scale applies to all the top panels and indicates counts from zero to $p$ where $p$ equals 3200, 1600, and 1600 for (a) to (c), respectively, and 800 for (g).

$\mathrm{MoS}_{2}$, for example, ranges from 3.5 to $6.0 \AA$ for photon energies from 70 to $170 \mathrm{eV}$, respectively, [50-52] comparable to the layer thickness of $\operatorname{ReSe}_{2}$ ( $c$ axis $6.7 \AA$ ), and a simple estimate based on the universal curve [53] gives $5.5 \AA$ at 100 eV. Despite this, Fig. 3 and Fig. 4 demonstrate systematic changes in the $\Gamma-M$ valence-band edge as a function of the number of layers. Overall, our nano-ARPES data reveals valence-band states that are clearly dependent on interlayer hopping interactions and must be representative of the body of the flake. We do not observe electronic states confined to individual layers except in the case of the $1 \mathrm{~L}$ sample itself. This is in agreement with studies of bulk and few-layer $\mathrm{ReS}_{2}[54,55]$. Thus, although the inter-layer interactions of $\mathrm{ReSe}_{2}$ are weak, very similar to $\mathrm{ReS}_{2}$ [32,41] (which shows a higher interlayer resistivity than typical TMDs [56]), they are certainly nonnegligible [57]. To compare, the interlayer coupling-induced $k_{z}$ dispersion of the highest valence band for $k_{x}=k_{y}=0$ in bulk $\mathrm{ReSe}_{2}$ is $\sim 100 \mathrm{meV}$ [25] while it is $\sim 800 \mathrm{meV}$ in $\mathrm{MoSe}_{2}$ and $\sim 1 \mathrm{eV}$ in $\mathrm{MoS}_{2}$ [58].

Recently, the surface sensitivity of ARPES has been applied to reveal hidden spin polarization [59] in centrosymmetric bulk TMDs, for example, $\mathrm{WSe}_{2}[60,61], \mathrm{MoS}_{2}$ [62], $\mathrm{NbSe}_{2}$ [63], and $\mathrm{PtSe}_{2}$ [64]. In the nomenclature of spinpolarisation effects introduced by Zhang et al. [59], D-1 signifies a spin polarization arising from conventional bulk Dresselhaus inversion asymmetry and D-2 implies localised Dresselhaus spin polarizations compensated by their opposites under bulk inversion symmetry. The case of $\mathrm{MoS}_{2}$ was considered theoretically [65]; $2 \mathrm{H}-\mathrm{MoS}_{2}$ is a system where individual D-1 layers interact (weakly) to give D-2 behavior in bulk. Surface-sensitive techniques such as STM or ARPES can probe the top layer of a bulk crystal and can reveal its D-1 nature. However, once again, $\mathrm{ReX}_{2}$ proves to be an untypical member of the TMD family, because its structure contains a center of inversion at the midpoint of each diamond of four Re atoms. On symmetry grounds, therefore, even a monolayer is only expected to show D-2 behavior. The opposing spin polarisations reside on Re atoms located close to one another in the same layer, meaning that the near single-layer sensitivity of ARPES cannot resolve individual D-1 contributions. This is borne out by the lack of observed spin-orbit splittings here or in any earlier ARPES data on bulk or few-layer $\mathrm{ReX}_{2}$ $[21,25,47,54,55,66]$.

\section{In-plane anisotropy and interlayer coupling}

In order to better understand the anisotropic change in the bandwidth of the top valence band as a function of the number of layers, we investigated the orbital character of the valence band of monolayer and bulk $\mathrm{ReSe}_{2}$ using density functional theory calculations and show the key results for monolayer in Fig. 5 (the complete set of projections for the monolayer as well as bulk are presented in Sec. S4 within the Supplemental Material [22]). In these calculations, we have neglected the spin-orbit coupling so that the atomic wave functions are purely orbital angular momentum states (this is acceptable because inversion symmetry, which forbids any band spin splitting is present for all crystal thicknesses, 

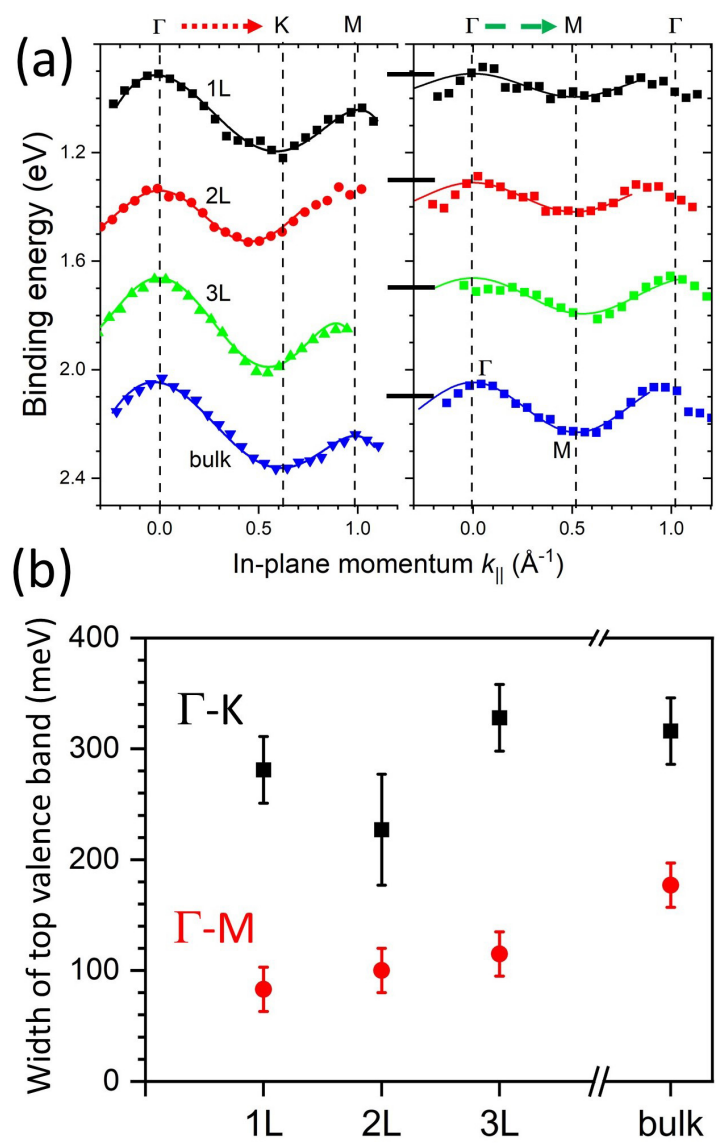

FIG. 4. Comparison of the measured $\mathrm{ReSe}_{2}$ valence-band dispersion along $\Gamma-M$ (as indicated by the green dashed arrow defined in Fig. 1) for mono-, bi-, and tri-layer flakes (labeled 1L, 2L, and $3 \mathrm{~L}$, respectively) and bulk; (a) band-edge positions determined from an EDC fit at each wave vector, with fits to each band edge to obtain the bandwidth. The curves for $2 \mathrm{~L}, 3 \mathrm{~L}$, and bulk samples are displaced vertically by $0.4 \mathrm{eV}$ from each other for clarity (the same shifts were applied to the $\Gamma-M$ and corresponding $\Gamma-K$ curves and the $1 \mathrm{~L}$ curves are unshifted); (b) summary of the bandwidths obtained in $\Gamma-M$ (red circles) and $\Gamma-K$ (black squares) directions for monolayer, bilayer, trilayer, and bulk $\mathrm{ReSe}_{2}$.

even monolayer). For this discussion, $x, y$ are defined as the directions along and normal to the Re chains respectively and $z$ is normal to the layer, as in Fig. 5(a).

In Fig. 5(b), we show the valence-band dispersion of monolayer $\operatorname{ReSe}_{2}$ along the directions $\Gamma-K$ and $\Gamma-M$ (calculated at the scalar relativistic GGA level) projected onto Se $p$ and $\operatorname{Re} d$ orbitals with the respective amplitudes shown using red and blue false color scales and white indicating an equal admixture. In general, the $\operatorname{Re} d$ states dominate in the conduction bands while the Se $p$ orbitals prevail on the valence-band side. However, the top valence band defies this simple picture: states in the vicinity of $\Gamma$ are built mainly of the Re $d$ orbitals while those around $K$ of Se $p$ orbitals. Along $\Gamma-M$, the $\operatorname{Re} d$-character band forming the valenceband maximum around $\Gamma$ crosses the second band made of a mixture of $\operatorname{Re} d$ and Se $p$ orbitals so that the highest energy states in this direction contain comparable transition metal and chalcogen contributions. We suggest it is this difference between the orbital composition of the top valence band in the directions along $(\Gamma-K)$ and perpendicular $(\Gamma-M)$ to the rhenium chains that leads to the unique coupling between in-plane anisotropy of this band and the crystal thickness. The sandwich-like nature of TMD monolayers means that chalcogen orbitals are more involved in, and hence affected by, the interlayer interaction than the rhenium orbitals. Hence, while states in the $\Gamma-M$ direction are significantly affected by the presence of other layers in thicker crystals which is reflected by the growing dispersion in Fig. 4(b), only a small part of states along $\Gamma-K$ responds to interlayer coupling so that the bandwidth in this direction remains similar in flakes of different thicknesses. In a sense, this mechanism is similar to the one driving the direct-indirect band-gap transition in $2 \mathrm{H}$ TMDs like $\mathrm{MoS}_{2}$ [12-14] where interlayer coupling causes shifts of the highest valence band in the vicinity of $\Gamma$ (made of out-of-plane $\mathrm{S} p_{z}$ and $\mathrm{Mo} d_{z^{2}}$ orbitals) while the states around $K$ (formed by in-plane Mo $d$ orbitals) remain unaffected. In $\mathrm{ReSe}_{2}$ however, out-of-plane orbitals do not contribute to the highest valence band and so the distinction is rather between the atomic species than orbital symmetries.

We investigate the orbital contributions of Re and Se to the valence band in more detail in Figs. 5(c)-5(j). We note that the four selenium sites that are not related by symmetry make different contributions to the band structure and, of these, one can identify two main types of chalcogen site: those located on the Re diamonds, labeled atoms 3 and 4 in Fig. 5(a), and those bridging adjacent Re chains (atoms 5 and 6) which differ markedly. Evidence for the nonequivalence of these sites is also provided by Raman spectroscopy studies of $\mathrm{ReSe}_{2-x} \mathrm{~S}_{x}$ alloys, which showed that the substitution of sulfur on the different chalcogen sites yields different formation energies and vibrational frequencies [25] whilst high resolution electron microscopy suggests preferential occupation of the more stable sites by impurities [67]. Scanning tunneling microscopy (STM) and spectroscopy (STS) likewise show very clearly the nonequivalence of the four selenium sites in $\mathrm{ReSe}_{2}$ [68]. The projections of the valence-band states onto the $\mathrm{Se} p$ orbitals, Figs. 5(c) and 5(d), show that it is the $p_{x}$ orbitals of Se sites 4 (and 3, see Fig. S15 within the Supplemental Material [22]) that contribute to the flat VBM in the $\Gamma-M$ direction. As seen in Figs. 5(d) and 5(h), the contribution of Se $p_{x}$ of sites 5 (and 6, Fig. S15 [22]) to the valence-band edge is less significant. From the coordinate system shown in Fig. 5(a), it is clear that the $p_{x}$ states of sites 3 and 4 are polarized along the Re chains and are spatially localized above and below them. The interaction between the orbitals of Se sites 3 and 4 on neighboring chains is minimal and this gives rise to a near-absence of dispersion in the $\Gamma-M(y)$ direction in the monolayer. The dispersion along the $\Gamma-K$ (x) direction is generated predominantly by $\operatorname{Re} d_{x^{2}-y^{2}}$ and $d_{z y}$ orbitals, Figs. 5(i) and 5(j).

\section{SUMMARY}

We have demonstrated that in $\mathrm{ReSe}_{2}$ in-plane anisotropy is quite uniquely coupled to interlayer interaction so that decreasing the number of layers in the crystal decreases the dispersion in the direction perpendicular to the rhenium chains ( $\Gamma-M$ in reciprocal space). This implies increasing inter- 


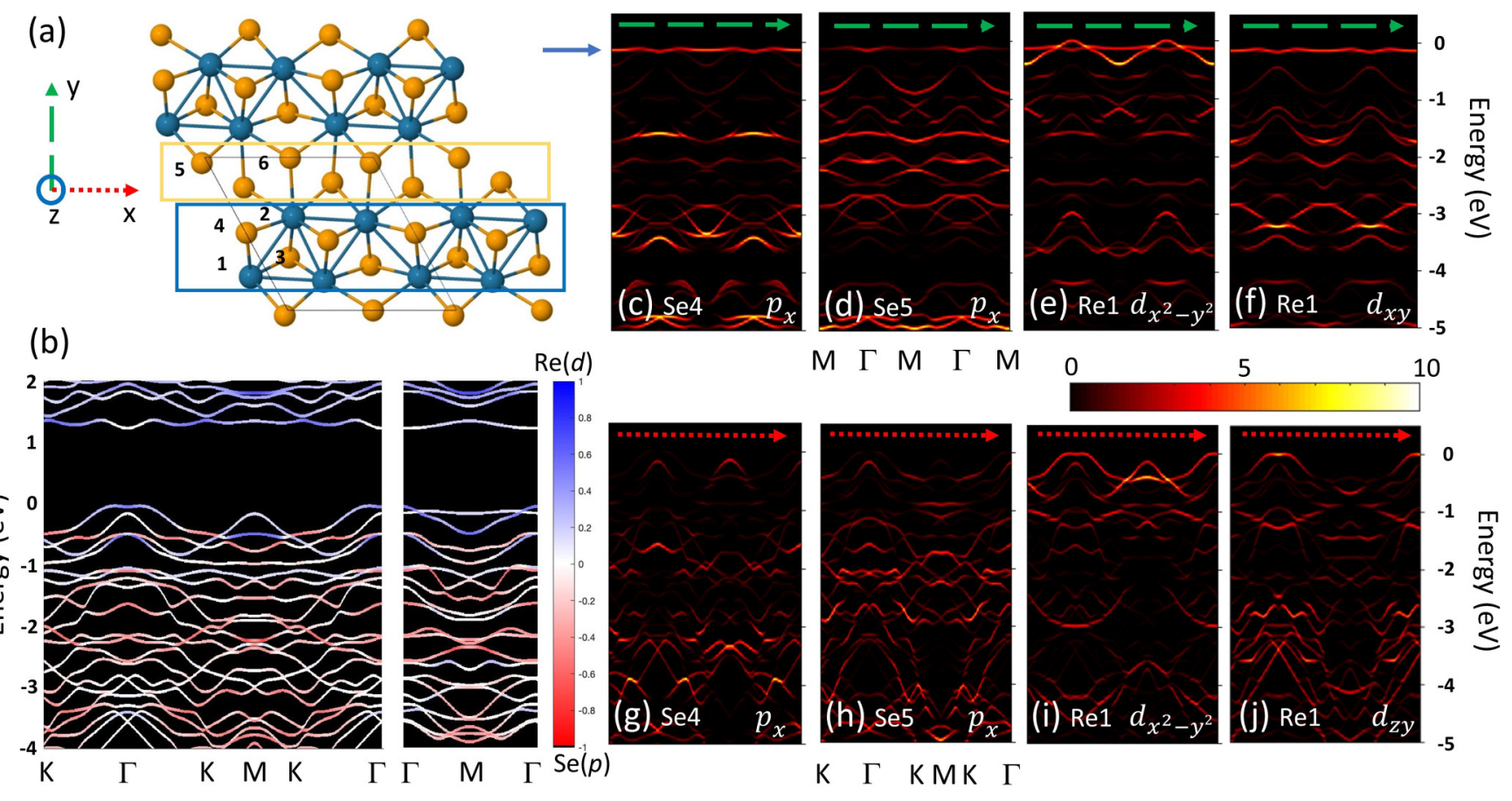

FIG. 5. The valence-band structure of monolayer $\mathrm{ReSe}_{2}$ analysed in terms of the atomic orbitals of rhenium and selenium. (a) top view of $\mathrm{ReSe}_{2}$ showing the numbering of the atoms: 1, 2 are Re, 3-6 are Se. Other atoms are related to these by symmetry. Rectangular boxes show the assignment of the chalcogen atoms to two families, those between rhenium chains (upper yellow box) and those above and below the chains (lower blue box). (b) Calculated valence-band dispersion of monolayer $\mathrm{ReSe}_{2}$ with states projected onto Se $p$ and Re $d$ orbitals (normalised amplitudes shown using the red-blue false color scale shown on the right; white indicates an equal admixture). [(c)-(j)] Calculated valence-band states projected onto the selected Re and Se atomic orbitals identified on each panel for the in-plane momentum directions $\Gamma-M$ and $\Gamma-K$ defined in Fig. 2 (reciprocal space directions are indicated by dashed green arrows and dotted red arrows, respectively, at the top of each panel). The top of the valence band is defined as the zero of energy and the blue arrow by panel (c) marks the neardispersionless state discussed in the text. The color scale in units of states per eV applying to panels (c)-(j) is shown below panels (e) and (f).

chain decoupling and a growing one-dimensional character of electronic states in this material. The extremely flat valenceband dispersion perpendicular to the Re chains implies that, in monolayer $\mathrm{ReSe}_{2}$, hole transport should be dominated by conduction along the direction of the Re chains and thus should be extremely anisotropic. No experimental test of this has yet been carried out, though it is established that the in-plane mobility is lowest perpendicular to the Re chains in bulk-like material. An anisotropy of about a factor of two between mobilities in the $a$ and $b$ directions was reported for few-layer $\mathrm{ReSe}_{2-x} \mathrm{~S}_{x}$ devices [69] but their reported number of layers was $\sim 5$, which as shown above is too thick to observe the present effects; furthermore, they observed $n$-type conductivity. Another study found an anisotropy ratio of $\sim 4$ for $n$-type conductivity in W-doped bulk material [70] in agreement with a recent ARPES study [71]. Several other studies on exfoliated material likewise used few-layer flakes down to $3 \mathrm{~L}$, or $n$-type material, and so do not provide a test of the present predictions [72-76]. There is only one report of measurements on $p$-type conductivity in a monolayer, where a hole mobility of $10 \mathrm{~cm}^{2} \mathrm{~V}^{-1} \mathrm{~s}^{-1}$ was found, but the anisotropy of the charge transport was apparently not investigated [76]. It is therefore a high priority to investigate transport in monolayer $\mathrm{ReSe}_{2}$ in suitably designed structures, which would ideally allow for investigation both of the predicted anisotropy but also electrostatic gating to ensure hole transport dominates. The quasi-one-dimensional nature of hole transport in $\mathrm{ReSe}_{2}$ offers the possibility of a momentum-selective filter or contact to other TMD layers in either lateral or vertical heterostructures.

Data created during the research for this paper are freely available from the University of Bath data archive [77].

\section{ACKNOWLEDGMENTS}

This work was supported by the Centre for Graphene Science of the Universities of Bath and Exeter and by the EPSRC (UK) under Grants No. EP/G036101, No. EP/M022188, and No. EP/P004830. L.S.H. and S.M.G. are supported by the Bath/Bristol Centre for Doctoral Training in Condensed Matter Physics, EPSRC Grant No. EP/L015544. M.M-K. acknowledges support from the University of Bath International Funding Scheme. We thank the SOLEIL synchrotron for the provision of beam time; work at SOLEIL was supported by EPSRC Grant No. EP/P004830/1. The present collaborative research was undertaken in the context of the Associated Unit MATINÉE of the Spanish Scientific Research Council (CSIC). Computational work was performed on the University of Bath's High Performance Computing Facility. 
[1] M. Bahramy, O. Clark, B.-J. Yang, J. Feng, L. Bawden, J. Riley, I. Marković, F. Mazzola, V. Sunko, and D. Biswas, Ubiquitous formation of bulk Dirac cones and topological surface states from a single orbital manifold in transition-metal dichalcogenides, Nat. Mater. 17, 21 (2018).

[2] K. F. Mak and J. Shan, Photonics and optoelectronics of 2D semiconductor transition metal dichalcogenides, Nat. Photonics 10, 216 (2016).

[3] Q. H. Wang, K. Kalantar-Zadeh, A. Kis, J. N. Coleman, and M. S. Strano, Electronics and optoelectronics of two-dimensional transition metal dichalcogenides, Nat. Nanotechnol. 7, 699 (2012).

[4] P. K. Nayak, Y. Horbatenko, S. Ahn, G. Kim, J.-U. Lee, K. Y. Ma, A. R. Jang, H. Lim, D. Kim, S. Ryu, H. Cheong, N. Park, and H. S. Shin, Probing evolution of twist-angle-dependent interlayer excitons in $\mathrm{MoSe}_{2} / \mathrm{WSe}_{2}$ van der Waals heterostructures, ACS Nano 11, 4041 (2017).

[5] Y. He, Y. Yang, Z. Zhang, Y. Gong, W. Zhou, Z. Hu, G. Ye, X. Zhang, E. Bianco, S. Lei et al., Strain-induced electronic structure changes in stacked van der Waals heterostructures, Nano Lett. 16, 3314 (2016).

[6] Y. Li, K.-A. N. Duerloo, K. Wauson, and E. J. Reed, Structural semiconductor-to-semimetal phase transition in twodimensional materials induced by electrostatic gating, Nat. Commun. 7, 10671 (2016).

[7] H.-J. Lamfers, A. Meetsma, G. Wiegers, and J. de Boer, The crystal structure of some rhenium and technetium dichalcogenides, J. Alloys Compd. 241, 34 (1996).

[8] M. Rahman, K. Davey, and S.-Z. Qiao, Advent of 2D rhenium disulfide $\left(\operatorname{ReS}_{2}\right)$ : Fundamentals to applications, Adv. Funct. Mater. 27, 1606129 (2017).

[9] L. Wang, Z. Sofer, J. Luxa, D. Sedmidubský, A. Ambrosi, and M. Pumera, Layered rhenium sulfide on free-standing three-dimensional electrodes is highly catalytic for the hydrogen evolution reaction: Experimental and theoretical study, Electrochem. Commun. 63, 39 (2016).

[10] J. A. Aliaga, T. Zepeda, J. F. Araya, F. ParaguayDelgado, E. Benavente, G. Alonso-Núñez, S. Fuentes, and G. González, Low-dimensional $\mathrm{ReS}_{2} / \mathrm{C}$ composite as effective hydrodesulfurization catalyst, Catalysts 7, 377 (2017).

[11] H. Zhao, Z. Dai, X. Xu, J. Pan, and J. Hu, Integrating semiconducting catalyst of $\mathrm{ReS}_{2}$ nanosheets into p-silicon photocathode for enhanced solar water reduction, ACS Appl. Mater. Interfaces 10, 23074 (2018).

[12] A. Splendiani, L. Sun, Y. Zhang, T. Li, J. Kim, C.-Y. Chim, G. Galli, and F. Wang, Emerging photoluminescence in monolayer $\mathrm{MoS}_{2}$, Nano Lett. 10, 1271 (2010).

[13] W. Jin, P.-C. Yeh, N. Zaki, D. Zhang, J. T. Sadowski, A. AlMahboob, A. M. van Der Zande, D. A. Chenet, J. I. Dadap, I. P. Herman, P. Sutter, J. Hone, and R. M. Osgood, Jr., Direct Measurement of the Thickness-Dependent Electronic Band Structure of $\mathrm{MoS}_{2}$ Using Angle-Resolved Photoemission Spectroscopy, Phys. Rev. Lett. 111, 106801 (2013).

[14] Y. Zhang, T.-R. Chang, B. Zhou, Y.-T. Cui, H. Yan, Z. Liu, F. Schmitt, J. Lee, R. Moore, Y. Chen et al., Direct observation of the transition from indirect to direct bandgap in atomically thin epitaxial $\mathrm{MoSe}_{2}$, Nat. Nanotechnol. 9, 111 (2014).
[15] C. S. Jung, F. Shojaei, K. Park, J. Y. Oh, H. S. Im, D. M. Jang, J. Park, and H. S. Kang, Red-to-ultraviolet emission tuning of two-dimensional gallium sulfide/selenide, ACS Nano 9, 9585 (2015).

[16] Z. Ben Aziza, D. Pierucci, H. Henck, M. G. Silly, C. David, M. Yoon, F. Sirotti, K. Xiao, M. Eddrief, J.-C. Girard, and A. Ouerghi, Tunable quasiparticle band gap in few-layer GaSe/graphene van der Waals heterostructures, Phys. Rev. B 96, 035407 (2017).

[17] M. J. Hamer, J. Zultak, A. V. Tyurnina, V. Zólyomi, D. Terry, A. Barinov, A. Garner, J. Donoghue, A. P. Rooney, V. Kandyba et al., Indirect to direct gap crossover in two-dimensional InSe revealed by angle-resolved photoemission spectroscopy, ACS Nano 13, 2136 (2019).

[18] H. Yuan, X. Liu, F. Afshinmanesh, W. Li, G. Xu, J. Sun, B. Lian, A. G. Curto, G. Ye, Y. Hikita, Z. Shen, S.-C. Zhang, X. Chen, M. Brongersma, H. Y. Hwang, and Y. Cui, Polarization-sensitive broadband photodetector using a black phosphorus vertical $\mathrm{p}-\mathrm{n}$ junction, Nat. Nanotechnol. 10, 707 (2015).

[19] A. Arora, J. Noky, M. Drüppel, B. Jariwala, T. Deilmann, R. Schneider, R. Schmidt, O. Del Pozo-Zamudio, T. Stiehm, A. Bhattacharya et al., Highly anisotropic in-plane excitons in atomically thin and bulklike 1T'- $\mathrm{ReSe}_{2}$, Nano Lett. 17, 3202 (2017).

[20] M. Kertesz and R. Hoffmann, Octahedral vs trigonal-prismatic coordination and clustering in transition-metal dichalcogenides, J. Am. Chem. Soc. 106, 3453 (1984).

[21] B. K. Choi, S. Ulstrup, S. M. Gunasekera, J. Kim, S. Y. Lim, L. Moreschini, J. S. Oh, S.-H. Chun, C. Jozwiak, A. Bostwick et al., Visualizing orbital content of electronic bands in anisotropic 2D semiconducting $\mathrm{ReSe}_{2}$, ACS Nano 14, 7880 (2020).

[22] See Supplemental Material at http://link.aps.org/supplemental/ 10.1103/PhysRevB.104.035421 for optical and AFM images of monolayer and few-layer flakes; photoemission energy distribution curves for monolayer $\mathrm{ReSe}_{2}$; calculated VB dispersion in the $\Gamma-M$ direction for few-layer and bulk $\mathrm{ReSe}_{2}$; measured VB dispersions of few-layer $\mathrm{ReSe}_{2}$ and their second derivatives; comparison of calculated and experimental dispersions of $\mathrm{ReSe}_{2}$; projections of calculated VB dispersion onto Se $p$ - and Re $d$-orbitals in $\Gamma-K$ and $\Gamma-M$ directions.

[23] A. Castellanos-Gomez, M. Buscema, R. Molenaar, V. Singh, L. Janssen, H. S. Van Der Zant, and G. A. Steele, Deterministic transfer of two-dimensional materials by all-dry viscoelastic stamping, 2D Materials 1, 011002 (2014).

[24] J. J. Yeh and I. Lindau, Atomic subshell photoionization cross sections and asymmetry parameters: $1 \leqslant z \leqslant 103$, At. Data Nucl. Data Tables 32, 1 (1985).

[25] L. S. Hart, J. L. Webb, S. Dale, S. J. Bending, M. MuchaKruczynski, D. Wolverson, C. Chen, J. Avila, and M. C. Asensio, Electronic bandstructure and van der waals coupling of $\mathrm{ReSe}_{2}$ revealed by high-resolution angle-resolved photoemission spectroscopy, Sci. Rep. 7, 5145 (2017).

[26] P. Giannozzi, S. Baroni, N. Bonini, M. Calandra, R. Car, C. Cavazzoni, D. Ceresoli, G. L. Chiarotti, M. Cococcioni, I. Dabo et al., QUANTUM ESPRESSO: A modular and open-source software project for quantum simulations of materials, J. Phys.: Condens. Matter 21, 395502 (2009).

[27] P. E. Blöchl, Projector augmented-wave method, Phys. Rev. B 50, 17953 (1994). 
[28] G. Kresse and D. Joubert, From ultrasoft pseudopotentials to the projector augmented-wave method, Phys. Rev. B 59, 1758 (1999).

[29] H. J. Monkhorst and J. D. Pack, Special points for Brillouinzone integrations, Phys. Rev. B 13, 5188 (1976).

[30] D. Wolverson, S. Crampin, A. S. Kazemi, A. Ilie, and S. J. Bending, Raman spectra of monolayer, few-layer, and bulk $\mathrm{ReSe}_{2}$ : An anisotropic layered semiconductor, ACS Nano 8, 11154 (2014).

[31] S. M. Gunasekera, D. Wolverson, L. S. Hart, and M. Mucha-Kruczynski, Electronic band structure of rhenium dichalcogenides, J. Electron. Mater. 47, 4314 (2018).

[32] H. Zhao, J. Wu, H. Zhong, Q. Guo, X. Wang, F. Xia, L. Yang, P. Tan, and H. Wang, Interlayer interactions in anisotropic atomically thin rhenium diselenide, Nano Res. 8, 3651 (2015).

[33] H.-X. Zhong, S. Gao, J.-J. Shi, and L. Yang, Quasiparticle band gaps, excitonic effects, and anisotropic optical properties of the monolayer distorted $1 T$ diamond-chain structures $\mathrm{ReS}_{2}$ and $\mathrm{ReSe}_{2}$, Phys. Rev. B 92, 115438 (2015).

[34] S. Grimme, J. Antony, S. Ehrlich, and H. Krieg, A consistent and accurate $\mathrm{ab}$ initio parametrization of density functional dispersion correction (DFT-D) for the 94 elements H-Pu, J. Chem. Phys. 132, 154104 (2010).

[35] L. Hart, S. Dale, S. Hoye, J. L. Webb, and D. Wolverson, Rhenium dichalcogenides: Layered semiconductors with two vertical orientations, Nano Lett. 16, 1381 (2016).

[36] A. McCreary, J. R. Simpson, Y. Wang, D. Rhodes, K. Fujisawa, L. Balicas, M. Dubey, V. H. Crespi, M. Terrones, and A. R. Hight Walker, Intricate resonant raman response in anisotropic $\mathrm{ReS}_{2}$, Nano Lett. 17, 5897 (2017).

[37] E. Liu, Y. Fu, Y. Wang, Y. Feng, H. Liu, X. Wan, W. Zhou, B. Wang, L. Shao, C.-H. Ho et al., Integrated digital inverters based on two-dimensional anisotropic $\mathrm{ReS}_{2}$ field-effect transistors, Nat. Commun. 6, 6991 (2015).

[38] D. A. Chenet, O. B. Aslan, P. Y. Huang, C. Fan, A. M. van der Zande, T. F. Heinz, and J. C. Hone, In-plane anisotropy in mono- and few-layer $\mathrm{ReS}_{2}$ probed by raman spectroscopy and scanning transmission electron microscopy, Nano Lett. 15, 5667 (2015).

[39] B. Jariwala, A. Thamizhavel, and A. Bhattacharya, $\mathrm{ReSe}_{2}$ : A reassessment of crystal structure and thermal analysis, J. Phys. D 50, 044001 (2016).

[40] P. Blake, E. Hill, A. Castro Neto, K. Novoselov, D. Jiang, R. Yang, T. Booth, and A. Geim, Making graphene visible, Appl. Phys. Lett. 91, 063124 (2007).

[41] S. Tongay, H. Sahin, C. Ko, A. Luce, W. Fan, K. Liu, J. Zhou, Y.-S. Huang, C.-H. Ho, J. Yan et al., Monolayer behaviour in bulk $\mathrm{ReS}_{2}$ due to electronic and vibrational decoupling, Nat. Commun. 5, 3252 (2014).

[42] W. Smekal, W. S. M. Werner, and C. J. Powell, Simulation of electron spectra for surface analysis (SESSA): A novel software tool for quantitative Auger-electron spectroscopy and $\mathrm{X}$-ray photoelectron spectroscopy, Surf. Interface Anal. 37, 1059 (2005).

[43] C. H. Ho, Y. S. Huang, J. L. Chen, T. E. Dann, and K. K. Tiong, Electronic structure of $\mathrm{ReS}_{2}$ and $\mathrm{ReSe}_{2}$ from first-principles calculations, photoelectron spectroscopy, and electrolyte electroreflectance, Phys. Rev. B 60, 15766 (1999).

[44] C. H. Ho, Y. S. Huang, K. K. Tiong, and P. C. Liao, Absorptionedge anisotropy in $\mathrm{ReS}_{2}$ and $\mathrm{ReSe}_{2}$ layered semiconductors, Phys. Rev. B 58, 16130 (1998).

[45] C.-H. Ho, H. Lee, and C. Wu, Polarization sensitive behaviour of the band-edge transitions in $\mathrm{ReS}_{2}$ and $\mathrm{ReSe}_{2}$ layered semiconductors, J. Phys.: Condens. Matter 16, 5937 (2004).

[46] C. Ho, M. Hsieh, C. Wu, Y. Huang, and K. Tiong, Dichroic optical and electrical properties of rhenium dichalcogenides layer compounds, J. Alloys Compd. 442, 245 (2007).

[47] P. Eickholt, J. Noky, E. F. Schwier, K. Shimada, K. Miyamoto, T. Okuda, C. Datzer, M. Drüppel, P. Krüger, M. Rohlfing, and M. Donath, Location of the valence band maximum in the band structure of anisotropic 1T'- $\mathrm{ReSe}_{2}$, Phys. Rev. B 97, 165130 (2018).

[48] N. R. Wilson, P. V. Nguyen, K. Seyler, P. Rivera, A. J. Marsden, Z. P. L. Laker, G. C. Constantinescu, V. Kandyba, A. Barinov, N. D. M. Hine et al., Determination of band offsets, hybridization, and exciton binding in 2D semiconductor heterostructures, Sci. Adv. 3, e1601832 (2017).

[49] H. Coy Diaz, J. Avila, C. Chen, R. Addou, M. C. Asensio, and M. Batzill, Direct observation of interlayer hybridization and Dirac relativistic carriers in graphene/ $\mathrm{MoS}_{2}$ van der Waals heterostructures, Nano Lett. 15, 1135 (2015).

[50] S. W. Han, G.-B. Cha, E. Frantzeskakis, I. Razado-Colambo, J. Avila, Y. S. Park, D. Kim, J. Hwang, J. S. Kang, S. Ryu et al., Band-gap expansion in the surface-localized electronic structure of $\mathrm{MoS}_{2}$ (0002), Phys. Rev. B 86, 115105 (2012).

[51] J. R. Lince, T. B. Stewart, M. M. Hills, P. D. Fleischauer, J. A. Yarmoff, and A. Taleb-Ibrahimi, Chemical effects of Ne+ bombardment on the $\mathrm{MoS}_{2}(0001)$ surface studied by high-resolution photoelectron spectroscopy, Surf. Sci. 210, 387 (1989).

[52] S. Tanuma, C. J. Powell, and D. R. Penn, Proposed formula for electron inelastic mean free paths based on calculations for 31 materials, Surf. Sci. 192, L849 (1987).

[53] M. P. Seah and W. A. Dench, Quantitative electron spectroscopy of surfaces: A standard data base for electron inelastic mean free paths in solids, Surf. Interface Anal. 1, 2 (1979).

[54] D. Biswas, A. M. Ganose, R. Yano, J. Riley, L. Bawden, O. Clark, J. Feng, L. Collins-Mcintyre, M. Sajjad, W. Meevasana, T. K. Kim, M. Hoesch, J. E. Rault, T. Sasagawa, D. O. Scanlon, and P. D. C. King, Narrow-band anisotropic electronic structure of $\mathrm{ReS}_{2}$, Phys. Rev. B 96, 085205 (2017).

[55] M. Gehlmann, I. Aguilera, G. Bihlmayer, S. Nemšák, P. Nagler, P. Gospodarič, G. Zamborlini, M. Eschbach, V. Feyer, F. Kronast et al., Direct observation of the band gap transition in atomically thin $\operatorname{ReS}_{2}$, Nano Lett. 17, 5187 (2017).

[56] B. C. Lee, J. Na, J. H. Choi, H. Ji, G.-T. Kim, and M.-K. Joo, Probing distinctive electron conduction in multilayer rhenium disulfide, Adv. Mater. 31, 1805860 (2019).

[57] J. P. Echeverry and I. C. Gerber, Theoretical investigations of the anisotropic optical properties of distorted $1 \mathrm{~T}-\mathrm{ReS}_{2}$ and $\mathrm{ReSe}_{2}$ monolayers, bilayers, and in the bulk limit, Phys. Rev. B 97, 075123 (2018).

[58] T. Böker, R. Severin, A. Müller, C. Janowitz, R. Manzke, D. Voß, P. Krüger, A. Mazur, and J. Pollmann, Band structure of $\mathrm{MoS}_{2}, \mathrm{MoSe}_{2}$, and $\alpha-\mathrm{MoTe}_{2}$ : Angle-resolved photoelec- 
tron spectroscopy and ab initio calculations, Phys. Rev. B 64, 235305 (2001).

[59] X. Zhang, Q. Liu, J.-W. Luo, A. J. Freeman, and A. Zunger, Hidden spin polarization in inversion-symmetric bulk crystals, Nat. Phys. 10, 387 (2014).

[60] J. M. Riley, F. Mazzola, M. Dendzik, M. Michiardi, T. Takayama, L. Bawden, C. Granerød, M. Leandersson, T. Balasubramanian, M. Hoesch et al., Direct observation of spinpolarized bulk bands in an inversion-symmetric semiconductor, Nat. Phys. 10, 835 (2014).

[61] R. Bertoni, C. W. Nicholson, L. Waldecker, H. Hubener, C. Monney, U. DeGiovannini, M. Puppin, M. Hoesch, E. Springate, R. T. Chapman, C. Cacho, M. Wolf, A. Rubio, and R. Ernstorfer, Generation and Evolution of Spin-, Valley-, and Layer-Polarized Excited Carriers in Inversion-Symmetric $\mathrm{WSe}_{2}$, Phys. Rev. Lett. 117, 277201 (2016).

[62] M. Gehlmann, I. Aguilera, G. Bihlmayer, E. Młyńczak, M. Eschbach, S. Döring, P. Gospodarič, S. Cramm, B. Kardynał, L. Plucinski, S. Blügel, and C. M. Schneider, Quasi 2D electronic states with high spin-polarization in centrosymmetric $\mathrm{MoS}_{2}$ bulk crystals, Sci. Rep. 6, 26197 (2016).

[63] L. Bawden, S. Cooil, F. Mazzola, J. Riley, L. Collins-McIntyre, V. Sunko, K. Hunvik, M. Leandersson, C. Polley, and T. Balasubramanian, Spin-valley locking in the normal state of a transition-metal dichalcogenide superconductor, Nat. Commun. 7, 11711 (2016).

[64] W. Yao, E. Wang, H. Huang, K. Deng, M. Yan, K. Zhang, K. Miyamoto, T. Okuda, L. Li, Y. Wang et al., Direct observation of spin-layer locking by local Rashba effect in monolayer semiconducting $\mathrm{PtSe}_{2}$ film, Nat. Commun. 8, 14216 (2017).

[65] Q. Liu, X. Zhang, H. Jin, K. Lam, J. Im, A. J. Freeman, and A. Zunger, Search and design of nonmagnetic centrosymmetric layered crystals with large local spin polarization, Phys. Rev. B 91, 235204 (2015).

[66] J. L. Webb, L. S. Hart, D. Wolverson, C. Chen, J. Avila, and M. C. Asensio, Electronic band structure of $\operatorname{ReS}_{2}$ by high- resolution angle-resolved photoemission spectroscopy, Phys. Rev. B 96, 115205 (2017).

[67] W. Wen, J. Lin, K. Suenaga, Y. Guo, Y. Zhu, H.-P. Hsu, and L. Xie, Preferential S/Se occupation in an anisotropic $\operatorname{ReS}_{2-x} \mathrm{Se}_{2 x}$ monolayer alloy, Nanoscale 9, 18275 (2017).

[68] M. Hong, X. Zhou, N. Gao, S. Jiang, C. Xie, L. Zhao, Y. Gao, Z. Zhang, P. Yang, Y. Shi et al., Identifying the non-identical outermost selenium atoms and invariable band gaps across the grain boundary of anisotropic rhenium diselenide, ACS Nano 12, 10095 (2018).

[69] F. C. Liu, S. J. Zheng, A. Chaturvedi, V. Zolyomi, J. D. Zhou, Q. D. Fu, C. Zhu, P. Yu, Q. S. Zeng, N. D. Drummond et al., Optoelectronic properties of atomically thin ReSSe with weak interlayer coupling, Nanoscale 8, 5826 (2016).

[70] S. Hu, C. Liang, K. Tiong, Y. Huang, and Y. Lee, Electrical anisotropy of W-doped $\mathrm{ReSe}_{2}$ crystals, J. Electrochem. Soc. 153, J100 (2006).

[71] B. S. Kim, W. S. Kyung, J. D. Denlinger, C. Kim, and S. R. Park, Strong one-dimensional characteristics of hole-carriers in $\mathrm{ReS}_{2}$ and $\mathrm{ReSe}_{2}$, Sci. Rep. 9, 2730 (2019).

[72] C. M. Corbet, C. McClellan, A. Rai, S. S. Sonde, E. Tutuc, and S. K. Banerjee, Field effect transistors with current saturation and voltage gain in ultrathin $\operatorname{ReS}_{2}$, ACS Nano 9, 363 (2015).

[73] M. H. Ali, D.-H. Kang, and J.-H. Park, Rhenium diselenide $\left(\mathrm{ReSe}_{2}\right)$ infrared photodetector enhanced by (3-aminopropyl) trimethoxysilane (APTMS) treatment, Organic Electronics 53, 14 (2018).

[74] E. Zhang, P. Wang, Z. Li, H. Wang, C. Song, C. Huang, Z.G. Chen, L. Yang, K. Zhang, S. Lu et al., Tunable ambipolar polarization-sensitive photodetectors based on high-anisotropy $\mathrm{ReSe}_{2}$ nanosheets, ACS Nano 10, 8067 (2016).

[75] S. Yang, S. Tongay, Q. Yue, Y. Li, B. Li, and F. Lu, High-performance few-layer Mo-doped $\mathrm{ReSe}_{2}$ nanosheet photodetectors, Sci. Rep. 4, 5442 (2014).

[76] S. Yang, S. Tongay, Y. Li, Q. Yue, J.-B. Xia, S.-S. Li, J. Li, and S.-H. Wei, Layer-dependent electrical and optoelectronic responses of $\mathrm{ReSe}_{2}$ nanosheet transistors, Nanoscale 6, 7226 (2014).

[77] See, 10.15125/BATH-00521 